\title{
Generalized 2-absorbing and strongly generalized 2-absorbing second submodules
}

\author{
H. Ansari-Toroghy, F. Farshadifar*, \\ and S. Maleki-Roudposhti
}

\author{
Communicated by D. Simson
}

\begin{abstract}
Let $R$ be a commutative ring with identity. A proper submodule $N$ of an $R$-module $M$ is said to be a 2-absorbing submodule of $M$ if whenever $a b m \in N$ for some $a, b \in R$ and $m \in M$, then $a m \in N$ or $b m \in N$ or $a b \in\left(N:_{R} M\right)$. In [3], the authors introduced two dual notion of 2-absorbing submodules (that is, 2absorbing and strongly 2-absorbing second submodules) of $M$ and investigated some properties of these classes of modules. In this paper, we will introduce the concepts of generalized 2-absorbing and strongly generalized 2-absorbing second submodules of modules over a commutative ring and obtain some related results.
\end{abstract}

\section{Introduction}

Throughout this paper, $R$ will denote a commutative ring with identity and $\mathbb{Z}$ will denote the ring of integers.

Let $M$ be an $R$-module. A proper submodule $P$ of $M$ is said to be prime if for any $r \in R$ and $m \in M$ with $r m \in P$, we have $m \in P$ or $r \in\left(P:_{R} M\right)$ [14]. A non-zero submodule $S$ of $M$ is said to be second if for each $a \in R$, the homomorphism $S \stackrel{a}{\rightarrow} S$ is either surjective or zero [17]. In this case $\operatorname{Ann}_{R}(S)$ is a prime ideal of $R$. A proper submodule $N$ of $M$ is said to be completely irreducible if $N=\bigcap_{i \in I} N_{i}$, where $\left\{N_{i}\right\}_{i \in I}$

${ }^{*}$ The corresponding author.

2010 MSC: 13C13, 13C99.

Key words and phrases: second, generalized 2-absorbing second. 
is a family of submodules of $M$, implies that $N=N_{i}$ for some $i \in I$. It is easy to see that every submodule of $M$ is an intersection of completely irreducible submodules of $M$ [15].

Badawi gave a generalization of prime ideals in [9] and said such ideals 2-absorbing ideals. A proper ideal $I$ of $R$ is a 2-absorbing ideal of $R$ if whenever $a, b, c \in R$ and $a b c \in I$, then $a b \in I$ or $a c \in I$ or $b c \in I$. He proved that $I$ is a 2-absorbing ideal of $R$ if and only if whenever $I_{1}, I_{2}$, and $I_{3}$ are ideals of $R$ with $I_{1} I_{2} I_{3} \subseteq I$, then $I_{1} I_{2} \subseteq I$ or $I_{1} I_{3} \subseteq I$ or $I_{2} I_{3} \subseteq I$. In [10], the authors introduced the concept of 2-absorbing primary ideal which is a generalization of primary ideal. A proper ideal $I$ of $R$ is called a 2-absorbing primary ideal of $R$ if whenever $a, b, c \in R$ and $a b c \in I$, then $a b \in I$ or $a c \in \sqrt{I}$ or $b c \in \sqrt{I}$.

The authors in [13] and [16], extended the concept of 2-absorbing ideals to the concept of 2-absorbing submodules. A proper submodule $N$ of $M$ is called a 2-absorbing submodule of $M$ if whenever abm $\in N$ for some $a, b \in R$ and $m \in M$, then $a m \in N$ or $b m \in N$ or $a b \in\left(N:_{R} M\right)$.

In [3], the authors introduced two dual notion of 2-absorbing submodules (that is, 2-absorbing and strongly 2-absorbing second submodules) of $M$ and investigated some properties of these classes of modules. A non-zero submodule $N$ of $M$ is said to be a 2-absorbing second submodule of $M$ if whenever $a, b \in R, L$ is a completely irreducible submodule of $M$, and $a b N \subseteq L$, then $a N \subseteq L$ or $b N \subseteq L$ or $a b \in \operatorname{Ann}_{R}(N)$. A non-zero submodule $N$ of $M$ is said to be a strongly 2-absorbing second submodule of $M$ if whenever $a, b \in R, K$ is a submodule of $M$, and $a b N \subseteq K$, then $a N \subseteq K$ or $b N \subseteq K$ or $a b \in \operatorname{Ann}_{R}(N)$.

The purpose of this paper is to introduce the concepts of generalized and strongly generalized 2-absorbing second submodules of an $R$-module $M$ as generalizations of 2-absorbing and strongly 2-absorbing second submodules of $M$ respectively, and provide some information concerning these new classes of modules.

\section{Main results}

Definition 2.1. We say that a non-zero submodule $N$ of an $R$-module $M$ is a generalized 2-absorbing second submodule or G2-absorbing second submodule of $M$ if whenever $a, b \in R, L$ is a completely irreducible submodule of $M$ and $a b N \subseteq L$, then $a \in \sqrt{\left(L:_{R} N\right)}$ or $b \in \sqrt{\left(L:_{R} N\right)}$ or $a b \in \operatorname{Ann}_{R}(N)$. By a generalized 2-absorbing second module, we mean a module which is a generalized 2-absorbing second submodule of itself. 
Example 2.2. Clearly every 2-absorbing second submodule is a $G 2$ absorbing second submodule. But the converse is not true in general as we will see in the Example 2.8.

We recall that an $R$-module $M$ is said to be a cocyclic module if $\operatorname{Soc}_{R}(M)$ is a large and simple submodule of $M$ [18]. (Here $\operatorname{Soc}_{R}(M)$ denotes the sum of all minimal submodules of $M$.). A submodule $L$ of $M$ is a completely irreducible submodule of $M$ if and only if $M / L$ is a cocyclic $R$-module [15].

Proposition 2.3. Let $N$ be a $G 2$-absorbing second submodule of an $R$-module $M$. Then we have the following.

(a) If $L$ is a completely irreducible submodule of $M$ such that $N \nsubseteq L$, then $\left(L:_{R} N\right)$ is a 2-absorbing primary ideal of $R$.

(b) If $M$ is a cocyclic module, then $\operatorname{Ann}_{R}(N)$ is a 2-absorbing primary ideal of $R$.

(c) If $\operatorname{Ann}_{R}(N)$ is a primary ideal of $R$, then $\left(L:_{R} N\right)$ is a primary ideal of $R$ for all completely irreducible submodule $L$ of $M$ such that $N \nsubseteq L L$.

Proof. (a) Since $N \nsubseteq L$, we have $\left(L:_{R} N\right) \neq R$. Let $a, b, c \in R$ and $a b c \in\left(L:_{R} N\right)$. Then $a b N \subseteq\left(L:_{M} c\right)$. Thus $a^{t} N \subseteq\left(L:_{M} c\right)$ for some positive integer $t$ or $b^{s} N \subseteq\left(L:_{M} c\right)$ for some positive integer $s$ or $a b N=0$ because by [7, 2.1], $\left(L:_{M} c\right)$ is a completely irreducible submodule of $M$. Therefore, $a c \in \sqrt{\left(L:_{R} N\right)}$ or $b c \in \sqrt{\left(L:_{R} N\right)}$ or $a b \in\left(L:_{R} N\right)$.

(b) Since $M$ is cocyclic, the zero submodule of $M$ is a completely irreducible submodule of $M$. Thus the result follows from part (a).

(c) Let $a, b \in R, L$ be a completely irreducible submodule of $M$ such that $N \nsubseteq L$, and $a b \in\left(L:_{R} N\right)$. Then $a^{t} N \subseteq L$ for some positive integer $t$ or $b^{s} N \subseteq L$ for some positive integer $s$ or $a b N=0$. If $a b N=0$, then by assumption, $a \in \sqrt{\operatorname{Ann}_{R}(N)}$ or $b N=0$. Thus in any cases we get that, $a \in \sqrt{\left(L:_{R} N\right)}$ or $b \in \sqrt{\left(L:_{R} N\right)}$.

Lemma 2.4. Let $I$ be an ideal of $R$ and $N$ be a $G 2$-absorbing second submodule of $M$. If $a \in R, L$ is a completely irreducible submodule of $M$, and $I a N \subseteq L$, then $a \in \sqrt{\left(L:_{R} N\right)}$ or $I \subseteq \sqrt{\left(L:_{R} N\right)}$ or $I a \subseteq \operatorname{Ann}_{R}(N)$.

Proof. Let $a \notin \sqrt{\left(L:_{R} N\right)}$ and $I a \nsubseteq \operatorname{Ann}_{R}(N)$. Then there exists $b \in I$ such that $a b N \neq 0$. Now as $N$ is a $G 2$-absorbing second submodule of $M$, $b a N \subseteq L$ implies that $b \in \sqrt{\left(L:_{R} N\right)}$. We show that $I \subseteq \sqrt{\left(L:_{R} N\right)}$. To see this, let $c$ be an arbitrary element of $I$. Then $(b+c) a N \subseteq L$. Hence, either $b+c \in \sqrt{\left(L:_{R} N\right)}$ or $(b+c) a \in \operatorname{Ann}_{R}(N)$. If $b+c \in \sqrt{\left(L:_{R} N\right)}$, then 
since $b \in \sqrt{\left(L:_{R} N\right)}$ we have $c \in \sqrt{\left(L:_{R} N\right)}$. If $(b+c) a \in \operatorname{Ann}_{R}(N)$, then $c a \notin \operatorname{Ann}_{R}(N)$, but $c a N \subseteq L$. Thus $c \in \sqrt{\left(L:_{R} N\right)}$. Hence, we conclude that $I \subseteq \sqrt{(L: R N)}$.

Theorem 2.5. Let $I$ and $J$ be two ideals of $R$ and $N$ be a G2-absorbing second submodule of $M$. If $L$ is a completely irreducible submodule of $M$ and $I J N \subseteq L$, then $I \subseteq \sqrt{\left(L:_{R} N\right)}$ or $J \subseteq \sqrt{\left(L:_{R} N\right)}$ or $I J \subseteq$ $\operatorname{Ann}_{R}(N)$.

Proof. Let $I \nsubseteq \sqrt{\left(L:_{R} N\right)}$ and $J \nsubseteq \sqrt{\left(L:_{R} N\right)}$. We show that $I J \subseteq$ $\operatorname{Ann}_{R}(N)$. Assume that $c \in I$ and $d \in J$. By assumption there exists $a \in I$ such that $a \notin \sqrt{\left(L:_{R} N\right)}$ but $a J N \subseteq L$. Now Lemma 2.4 shows that $a J \subseteq \operatorname{Ann}_{R}(N)$ and so $\left(I \backslash \sqrt{\left(L:_{R} N\right)}\right) J \subseteq \operatorname{Ann}_{R}(N)$. Similarly there exists $b \in\left(J \backslash \sqrt{\left(L:_{R} N\right)}\right)$ such that $I b \subseteq \operatorname{Ann}_{R}(N)$ and also $I\left(J \backslash \sqrt{\left(L:_{R} N\right)}\right) \subseteq \operatorname{Ann}_{R}(N)$. Thus we have $a b \in \operatorname{Ann}_{R}(N)$, ad $\in$ $\operatorname{Ann}_{R}(N)$ and $c b \in \operatorname{Ann}_{R}(N)$. As $a+c \in I$ and $b+d \in J$, we have $(a+c)(b+d) N \subseteq L$. Therefore, $a+c \in \sqrt{\left(L:_{R} N\right)}$ or $b+d \in \sqrt{\left(L:_{R} N\right)}$ or $(a+c)(b+d) \in \operatorname{Ann}_{R}(N)$. If $a+c \in \sqrt{\left(L:_{R} N\right)}$, then $c \notin \sqrt{\left(L:_{R} N\right)}$. Hence $c \in I \backslash \sqrt{\left(L:_{R} N\right)}$ which implies that $c d \in \operatorname{Ann}_{R}(N)$. Similarly if $b+d \in \sqrt{\left(L:_{R} N\right)}$, we can deduce that $c d \in \operatorname{Ann}_{R}(N)$. Finally if $(a+c)(b+d) \in \operatorname{Ann}_{R}(N)$, then $a b+a d+c b+c d \in \operatorname{Ann}_{R}(N)$ so that $c d \in \operatorname{Ann}_{R}(N)$. Therefore, $I J \subseteq \operatorname{Ann}_{R}(N)$.

Theorem 2.6. Let $N$ be a non-zero submodule of an $R$-module $M$. The following statements are equivalent:

(a) If $a b N \subseteq K$ for some $a, b \in R$ and a submodule $K$ of $M$, then $a \in \sqrt{\left(K:_{R} N\right)}$ or $b \in \sqrt{\left(K:_{R} N\right)}$ or $a b \in \operatorname{Ann}_{R}(N)$.

(b) If $I J N \subseteq K$ for some ideals $I$ and $J$ of $R$ and submodule $K$ of $M$, then $I \subseteq \sqrt{\left(K:_{R} N\right)}$ or $J \subseteq \sqrt{\left(K:_{R} N\right)}$ or $I J \subseteq \operatorname{Ann}_{R}(N)$.

Proof. $(a) \Rightarrow(b)$ The proof is similar to the proof of Theorem 2.5.

$(b) \Rightarrow(a)$ This is clear.

Definition 2.7. We say that a non-zero submodule $N$ of an $R$-module $M$ is a strongly generalized 2-absorbing second submodule or strongly G2absorbing second submodule of $M$ if satisfies the equivalent conditions of Theorem 2.6. By a strongly generalized 2-absorbing second module, we mean a module which is a strongly generalized 2-absorbing second submodule of itself.

Example 2.8. Clearly every strongly 2 -absorbing second submodule is a strongly $G 2$-absorbing second submodule. But the converse is not 
true in general. For example, for any prime integer $p$, let $M=\mathbb{Z}_{p^{\infty}}$ and $N=\left\langle 1 / p^{3}+\mathbb{Z}\right\rangle$. Then $N$ is a strongly $G 2$-absorbing second submodule which is not a strongly 2 -absorbing second submodule of $M$.

This is clear that every strongly $G 2$-absorbing second submodule is a $G 2$-absorbing second submodule. It is natural to ask the following question:

Question 2.9. Let $M$ be an $R$-module. Is every $G 2$-absorbing second submodule of $M$ a strongly $G 2$-absorbing second submodule of $M$ ?

Theorem 2.10. Let $N$ be a non-zero submodule of an Artinian $R$-module $M$. The following statements are equivalent:

(a) If $a b N \subseteq L_{1} \cap L_{2}$ for some $a, b \in R$ and completely irreducible submodules $L_{1}, L_{2}$ of $M$, then we have $a \in \sqrt{\left(L_{1} \cap L_{2}:_{R} N\right)}$ or $b \in \sqrt{\left(L_{1} \cap L_{2}:_{R} N\right)}$ or $a b \in \operatorname{Ann}_{R}(N)$.

(b) $N$ is a strongly G2-absorbing second submodule.

Proof. $(a) \Rightarrow(b)$. Assume that $a b N \subseteq K$ for some $a, b \in R$, a submodule $K$ of $M$, and $a b \nsubseteq \operatorname{Ann}_{R}(N)$. Since $M$ is Artinian, there exist completely irreducible submodules $L_{1}, L_{2}, \ldots, L_{n}$ of $M$ such that $K=\cap_{i=1}^{n} L_{i}$. Then for each $L_{i}(1 \leqslant i \leqslant n)$ either $a \in \sqrt{\left(L_{i}:_{R} N\right)}$ or $b \in \sqrt{\left(L_{i}:_{R} N\right)}$. If $a \in \sqrt{\left(L_{i}: R N\right)}$ for each $1 \leqslant i \leqslant n$, then

$$
\begin{aligned}
a \in \cap_{i=1}^{n} \sqrt{\left(L_{i}:_{R} N\right)} & =\sqrt{\cap_{i=1}^{n}\left(L_{i}:_{R} N\right)} \\
& =\sqrt{\left(\cap_{i=1}^{n} L_{i}:_{R} N\right)}=\sqrt{\left(K:_{R} N\right)} .
\end{aligned}
$$

Similarly, if $\left.b \in \sqrt{\left(L_{i}:_{R} N\right)}\right)$ for each $1 \leqslant i \leqslant n$, then we get that $b \in \sqrt{\left(K:_{R} N\right)}$. Now suppose that there exist $1 \leqslant i, j \leqslant n$ such that $a \notin \sqrt{\left(L_{i}:_{R} N\right)}$ and $b \notin \sqrt{\left(L_{j}:_{R} N\right)}$. Then $a \in \sqrt{\left(L_{j}:_{R} N\right)}$ and $b \in$ $\sqrt{\left(L_{i}:_{R} N\right)}$. Since $a b N \subseteq L_{i} \cap L_{j}$, we have either $a \in \sqrt{\left(L_{i} \cap L_{j}:_{R} N\right)}$ or $b \in \sqrt{\left(L_{i} \cap L_{j}:_{R} N\right)}$. If $a \in \sqrt{\left(L_{i} \cap L_{j}:_{R} N\right)}$, then $a \in \sqrt{\left(L_{i}:_{R} N\right)}$ which is a contradiction. Similarly from $b \in \sqrt{\left(L_{i} \cap L_{j}:_{R} N\right)}$ we get a contradiction.

$(b) \Rightarrow(a)$. This is clear.

Proposition 2.11. Let $M$ be an $R$-module. If either $N$ is a secondary submodule of $M$ or $\mathrm{N}$ is a finite sum of $p$-secondary submodules of $M$, then $N$ is strongly $G 2$-absorbing second submodule.

Proof. The first assertion is clear. Now the second assertion follows from $[11,3.1 .4]$. 
Lemma 2.12. Let $M$ be an $R$-module, $N \subseteq K$ be two submodules of $M$, and $K$ be a strongly $G 2$-absorbing second submodule of $M$. Then $K / N$ is a strongly $G 2$-absorbing second submodule of $M / N$.

Proof. This is straightforward.

Proposition 2.13. Let $N$ be a strongly $G 2$-absorbing second submodule of an $R$-module $M$. Then we have the following.

(a) $\operatorname{Ann}_{R}(N)$ is a 2-absorbing primary ideal of $R$.

(b) If $K$ is a submodule of $M$ such that $N \nsubseteq K$, then $\left(K:_{R} N\right)$ is a 2-absorbing primary ideal of $R$.

Proof. (a) Let $a, b, c \in R$ and $a b c \in \operatorname{Ann}_{R}(N)$. Then $a b N \subseteq a b N$ implies that $a^{t} N \subseteq a b N$ for some positive integer $t$ or $b^{s} N \subseteq a b N$ for some positive integer $s$ or $a b N=0$. If $a b N=0$, then we are done. If $a^{t} N \subseteq a b N$, then $(c a)^{t} N \subseteq c a^{t} N \subseteq c a b N=0$. Thus $c a \in \sqrt{\operatorname{Ann}_{R}(N)}$. In other case, we do the same.

(b) Let $a, b, c \in R$ and $a b c \in(K: R N)$. Then $a^{t} c N \subseteq K$ for some positive integer $t$ or $b^{s} c N \subseteq K$ for some positive integer $s$ or $a b N=0$. If $a^{t} c N \subseteq K$ or $b^{s} c N \subseteq K$, then $(a c)^{t} N \subseteq K$ or $(b c)^{s} N \subseteq K$ and so we are done. If $a b N=0$, then the result follows from part (a).

An $R$-module $M$ is said to be a comultiplication module if for every submodule $N$ of $M$ there exists an ideal $I$ of $R$ such that $N=\left(0:_{M} I\right)$, equivalently, for each submodule $N$ of $M$, we have $N=\left(0: M \operatorname{Ann}_{R}(N)\right)$ [5].

Corollary 2.14. Let $M$ be a comultiplication $R$-module. If $N$ is a strongly $G 2$-absorbing second submodule of $M$ such that $\sqrt{\operatorname{Ann}_{R}(N)}=\operatorname{Ann}_{R}(N)$, then $N$ is a strongly 2-absorbing second submodule of $M$.

Proof. By Proposition 2.13 (a), $\operatorname{Ann}_{R}(N)$ is a 2-absorbing primary ideal of $R$. Thus $\sqrt{\operatorname{Ann}_{R}(N)}=\operatorname{Ann}_{R}(N)$ is a 2-absorbing ideal of $R$ by [10, 2.2.]. Now the result follows from $[3,3.10]$.

A submodule $N$ of an $R$-module $M$ is said to be coidempotent if $N=\left(0: M \operatorname{Ann}_{R}(N)^{2}\right)$. Also, $M$ is said to be fully coidempotent if every submodule of $M$ is coidempotent [1]. Clearly, every fully coidempotent $R$-module is a comultiplication $R$-module.

Theorem 2.15. Let $R$ be a Noetherian ring and $N$ be a submodule of a fully coidempotent $R$-module $M$. Then we have the following.

(a) If $\operatorname{Ann}_{R}(N)$ is a 2-absorbing primary ideal of $R$, then $N$ is a strongly G2-absorbing second submodule of $M$. 
(b) If $M$ is a cocyclic module and $N$ is a G2-absorbing second submodule of $M$, then $N$ is a strongly G2-absorbing second submodule of $M$.

Proof. (a) Let $a, b \in R, K$ be a submodule of $M$, and $a b N \subseteq K$. Then we have $\operatorname{Ann}_{R}(K) a b N=0$. Now since $R$ is Noetherian, $\left(\operatorname{Ann}_{R}(K) a\right)^{t} N=0$ for some positive integer $t$ or $\left(\operatorname{Ann}_{R}(K) b\right)^{s} N=0$ for some positive integer $s$ or $a b N=0$ by [10, 2.18]. If $a b N=0$, we are done. If $\left(\operatorname{Ann}_{R}(K) a\right)^{t} N=0$ or $\left(\operatorname{Ann}_{R}(K) b\right)^{s} N=0$, then $\left(\operatorname{Ann}_{R}(K)\right)^{t} \subseteq \operatorname{Ann}_{R}\left(a^{t} N\right)$ or $\left(\operatorname{Ann}_{R}(K)\right)^{s} \subseteq$ $\operatorname{Ann}_{R}\left(b^{s} N\right)$. Hence, $a^{t} N \subseteq K$ or $b^{s} N \subseteq K$ since $M$ is a fully coidempotent $R$-module. Therefore, $N$ is a strongly $G 2$-absorbing second submodule of $M$.

(b) By Proposition 2.3, $\operatorname{Ann}_{R}(N)$ is a 2-absorbing primary ideal of $R$. Thus the result follows from part (a).

The following example shows that Theorem 2.15 (a) is not satisfied in general.

Example 2.16. By $[5,3.9]$, the $\mathbb{Z}$-module $\mathbb{Z}$ is not a comultiplication $\mathbb{Z}$-module and so it is not a fully coidempotent $\mathbb{Z}$-module. The submodule $N=p \mathbb{Z}$ of $\mathbb{Z}$, where $p$ is a prime number, is not strongly $G 2$-absorbing second submodule. But $\operatorname{Ann}_{\mathbb{Z}}(p \mathbb{Z})=0$ is a 2-absorbing primary ideal of $R$.

For a submodule $N$ of an $R$-module $M$ the second radical (or second socle) of $N$ is defined as the sum of all second submodules of $M$ contained in $N$ and it is denoted by $\sec (N)$ (or $\operatorname{soc}(N))$. In case $N$ does not contain any second submodule, the second radical of $N$ is defined to be (0) (see [12] and [2]).

Proposition 2.17. Let $M$ be a finitely generated comultiplication $R$ module. If $N$ is a strongly $G 2$-absorbing second submodule of $M$, then $\sec (N)$ is a strongly 2-absorbing second submodule of $M$.

Proof. Let $N$ be a strongly $G 2$-absorbing second submodule of $M$. By Proposition 2.13 (a), $\operatorname{Ann}_{R}(N)$ is a 2-absorbing primary ideal of $R$. Thus by [10, 2.2], $\sqrt{\operatorname{Ann}_{R}(N)}$ is a 2-absorbing ideal of $R$. By [6, 2.12], $\operatorname{Ann}_{R}(\sec (N))=\sqrt{\operatorname{Ann}_{R}(N)}$. Therefore, $\operatorname{Ann}_{R}(\sec (N))$ is a 2-absorbing ideal of $R$. Now the result follows from [3, 3.10].

A non-zero submodule $N$ of an $R$-module $M$ is a strongly 2-absorbing secondary submodule of $M$ if whenever $a, b \in R, K$ is a submodule of $M$ and $a b N \subseteq K$, then $a(\sec (N)) \subseteq K$ or $b(\sec (N)) \subseteq K$ or $a b \in \operatorname{Ann}_{R}(N)$ [4]. 
Theorem 2.18. Let $M$ be a comultiplication $R$-module and $N$ be a strongly G2-absorbing second submodule of $M$. Then $N$ is a strongly 2-absorbing secondary submodule of $M$.

Proof. Let $a, b \in R, K$ be a submodule of $M$, and $a b N \subseteq K$. Then we have $a^{t} N \subseteq K$ for some positive integer $t$ or $b^{s} N \subseteq K$ for some positive integer $s$ or $a b N=0$. If $a b N=0$, then we are done. Suppose that $a^{t} N \subseteq K$ for some positive integer $t$. As $M$ is a comultiplication $R$-module, $K=\left(0:_{M} I\right)$ for some ideal $I$ of $R$. Thus $I a^{t} N=0$. This implies that $I a \subseteq \sqrt{\operatorname{Ann}_{R}(N)}$. Thus

$$
\sec (N) \subseteq\left(0: M \sqrt{\operatorname{Ann}_{R}(N)}\right) \subseteq\left(0:_{M} I a\right)=\left(K:_{M} a\right) .
$$

Hence $a(\sec (N)) \subseteq K$, as needed.

Example 2.19. The submodule $N=p \mathbb{Z}$ of the $\mathbb{Z}$-module $M=\mathbb{Z}$, where $p$ is a prime number, is not a strongly $G 2$-absorbing second submodule. But as $\sec (p \mathbb{Z})=0$, we have $N$ is a strongly 2 -absorbing secondary submodule of $M$.

Theorem 2.20. Let $f: M \rightarrow M$ be a monomorphism of R-modules. Then we have the following.

(a) If $N$ is a strongly G2-absorbing second submodule of $M$, then $f(N)$ is a strongly G2-absorbing second submodule of $M$.

(b) If $N$ is a strongly G2-absorbing second submodule of $M$ and $N \subseteq$ $f(M)$, then $f^{-1}(N)$ is a strongly $G 2$-absorbing second submodule of $M$.

Proof. (a) Since $N \neq 0$ and $f$ is a monomorphism, we have $f(N) \neq 0$. Let $a, b \in R, K^{\prime}$ be a submodule of $M^{\prime}$, and $a b f(N) \subseteq \dot{K}$. Then $a b N \subseteq f^{-1}\left(K^{\prime}\right)$. As $N$ is strongly $G 2$-absorbing second submodule, $a^{t} N \subseteq f^{-1}(\dot{K})$ for some positive integer $t$ or $b^{s} N \subseteq f^{-1}(\bar{K})$ for some positive integer $s$ or $a b N=0$. Therefore,

$$
a^{t} f(N) \subseteq f\left(f^{-1}(\dot{K})\right)=f(M) \cap \dot{K} \subseteq K^{\prime}
$$

or

$$
b^{s} f(N) \subseteq f\left(f^{-1}\left(\dot{K}^{\prime}\right)\right)=f(M) \cap \dot{K} \subseteq K^{\prime}
$$

or $a b f(N)=0$, as needed.

(b) If $f^{-1}(N)=0$, then $f(M) \cap N=f\left(f^{-1}(N)\right)=f(0)=0$. Thus $N^{\prime}=0$, a contradiction. Therefore, $f^{-1}(\hat{N}) \neq 0$. Now let $a, b \in R, K$ be a submodule of $M$, and $a b f^{-1}(N) \subseteq K$. Then

$$
a b N=a b\left(f(M) \cap N^{\prime}\right)=a b f\left(f^{-1}\left(N^{\prime}\right)\right) \subseteq f(K) .
$$


As $N^{\prime}$ is strongly $G 2$-absorbing second submodule, $a^{t} N \subseteq f(K)$ for some positive integer $t$ or $b^{s} N \subseteq f(K)$ for some positive integer $s$ or $a b N=0$. Therefore, $a^{t} f^{-1}\left(N^{\prime}\right) \subseteq f^{-1}(f(K))=K$ or $b^{s} f^{-1}\left(N^{\prime}\right) \subseteq f^{-1}(f(K))=K$ or $a b f^{-1}\left(N^{\prime}\right)=0$ as desired.

Corollary 2.21. Let $M$ be an $R$-module and $N \subseteq K$ be two submodules of $M$. Then $N$ is a strongly $G 2$-absorbing second submodule of $K$ if and only if $N$ is a strongly $G 2$-absorbing second submodule of $M$.

Proof. This follows from Theorem 2.20 by using the natural monomorphism $K \rightarrow M$.

Let $N$ be a submodule of an $R$-module $M$. Then Corollary 2.21 shows that $N$ is a strongly $G 2$-absorbing second submodule of $M$ if and only if $N$ is a strongly $G 2$-absorbing second module.

Let $R_{i}$ be a commutative ring with identity and $M_{i}$ be an $R_{i}$-module, for $i=1,2$. Let $R=R_{1} \times R_{2}$. Then $M=M_{1} \times M_{2}$ is an $R$-module and each submodule of $M$ is in the form of $N=N_{1} \times N_{2}$ for some submodules $N_{1}$ of $M_{1}$ and $N_{2}$ of $M_{2}$.

Lemma 2.22. Let $R=R_{1} \times R_{2}$ and $M=M_{1} \times M_{2}$. Then $M_{i}$ is a fully coidempotent $R_{i}$-module, for $i=1,2$ if and only if $M$ is a fully coidempotent $R$-module.

Proof. First suppose that $M$ is a fully coidempotent $R$-module and $N_{1}$ is a submodule of an $R_{1}$-module $M_{1}$. Then $N=N_{1} \times 0$ is a submodule of $M$. Thus $N=\left(0::_{M} \operatorname{Ann}_{R}(N)^{2}\right)=\left(0:_{M_{1}} \operatorname{Ann}_{R_{1}}\left(N_{1}\right)^{2}\right) \times 0$. Hence $N_{1}=\left(0: M_{1} \operatorname{Ann}_{R_{1}}\left(N_{1}\right)^{2}\right)$. Therefore, $M_{1}$ is a fully coidempotent $R_{1^{-}}$ module. Similarly, $M_{2}$ is a fully coidempotent $R_{2}$-module. Conversely, let $N$ be a submodule of $M$. Then $N=N_{1} \times N_{2}$ for some submodules $N_{1}$ of $M_{1}$ and $N_{2}$ of $M_{2}$. By assumption, $N_{i}=\left(0: M_{i} \operatorname{Ann}_{R_{i}}\left(N_{i}\right)^{2}\right)$ for $i=1,2$. So

$$
N=\left(0:_{M_{1}} \operatorname{Ann}_{R_{1}}\left(N_{1}\right)^{2}\right) \times\left(0:_{M_{2}} \operatorname{Ann}_{R_{2}}\left(N_{2}\right)^{2}\right)=\left(0:_{M} \operatorname{Ann}_{R}(N)^{2}\right),
$$

as requested.

Theorem 2.23. Let $R=R_{1} \times R_{2}$ be a Noetherian ring and $M=M_{1} \times M_{2}$, where $M_{1}$ is a fully coidempotent $R_{1}$-module and $M_{2}$ is a fully coidempotent $R_{2}$-module. Then we have the following.

(a) A non-zero submodule $K_{1}$ of $M_{1}$ is a strongly G2-absorbing second submodule if and only if $N=K_{1} \times 0$ is a strongly G2-absorbing second submodule of $M$. 
(b) A non-zero submodule $K_{2}$ of $M_{2}$ is a strongly G2-absorbing second submodule if and only if $N=0 \times K_{2}$ is a strongly G2-absorbing second submodule of $M$.

(c) If $K_{1}$ is a secondary submodule of $M_{1}$ and $K_{2}$ is a secondary submodule of $M_{2}$, then $N=K_{1} \times K_{2}$ is a strongly $G 2$-absorbing second submodule of $M$.

Proof. (a) Let $K_{1}$ be a strongly $G 2$-absorbing second submodule of $M_{1}$. Then $\operatorname{Ann}_{R_{1}}\left(K_{1}\right)$ is a 2-absorbing primary ideal of $R_{1}$ by Proposition 2.13. Now since $\operatorname{Ann}_{R}(N)=\operatorname{Ann}_{R_{1}}\left(K_{1}\right) \times R_{2}$, we have $\operatorname{Ann}_{R}(N)$ is a 2-absorbing primary ideal of $R$ by [10,2.23]. Thus the result follows from Theorem 2.15 (a). Conversely, let $N=K_{1} \times 0$ be a strongly $G 2$-absorbing second submodule of $M$. Then $\operatorname{Ann}_{R}(N)=\operatorname{Ann}_{R_{1}}\left(K_{1}\right) \times R_{2}$ is a primary ideal of $R$ by Proposition 2.13. Thus $\operatorname{Ann}_{R_{1}}\left(K_{1}\right)$ is a primary ideal of $R_{1}$ by [10, 2.23]. Thus by Theorem 2.15 (a), $K_{1}$ is a strongly $G 2$-absorbing second submodule of $M_{1}$.

(b) This is proved similar to the part (a).

(c) Let $K_{1}$ be a secondary submodule of $M_{1}$ and $K_{2}$ be a secondary submodule of $M_{2}$. Then $\operatorname{Ann}_{R_{1}}\left(K_{1}\right)$ and $\operatorname{Ann}_{R_{2}}\left(K_{2}\right)$ are primary ideals of $R_{1}$ and $R_{2}$, respectively. Now since $\operatorname{Ann}_{R}(N)=\operatorname{Ann}_{R_{1}}\left(K_{1}\right) \times \operatorname{Ann}_{R_{2}}\left(K_{2}\right)$, we have $\operatorname{Ann}_{R}(N)$ is a 2-absorbing primary ideal of $R$ by [10, 2.23]. Thus the result follows from Theorem 2.15 (a).

Theorem 2.24. Let $R=R_{1} \times R_{2}$ be a Noetherian decomposable ring and $M=M_{1} \times M_{2}$ be a fully coidempotent $R$-module, where $M_{1}$ is an $R_{1}$-module and $M_{2}$ is an $R_{2}$-module. Suppose that $N=N_{1} \times N_{2}$ is a non-zero submodule of $M$. Then the following conditions are equivalent:

(a) $N$ is a strongly G2-absorbing second submodule of $M$;

(b) Either $N_{1}=0$ and $N_{2}$ is a strongly G2-absorbing second submodule of $M_{2}$ or $N_{2}=0$ and $N_{1}$ is a strongly $G 2$-absorbing second submodule of $M_{1}$ or $N_{1}, N_{2}$ are secondary submodules of $M_{1}, M_{2}$, respectively.

Proof. $(a) \Rightarrow(b)$. Let $N=N_{1} \times N_{2}$ be a strongly $G 2$-absorbing second submodule of $M$. Then $\operatorname{Ann}_{R}(N)=\operatorname{Ann}_{R_{1}}\left(N_{1}\right) \times \operatorname{Ann}_{R_{2}}\left(N_{2}\right)$ is a 2absorbing primary ideal of $R$ by Proposition 2.13. By [10, 2.23], we have $\operatorname{Ann}_{R_{1}}\left(N_{1}\right)=R_{1}$ and $\operatorname{Ann}_{R_{2}}\left(N_{2}\right)$ is a 2-absorbing primary ideal of $R_{2}$ or $\operatorname{Ann}_{R_{2}}\left(N_{2}\right)=R_{2}$ and $\operatorname{Ann}_{R_{1}}\left(N_{1}\right)$ is a 2-absorbing primary ideal of $R_{1}$ or $\operatorname{Ann}_{R_{1}}\left(N_{1}\right)$ and $\operatorname{Ann}_{R_{2}}\left(N_{2}\right)$ are primary ideals of $R_{1}$ and $R_{2}$, respectively. Suppose that $\operatorname{Ann}_{R_{1}}\left(N_{1}\right)=R_{1}$ and $\operatorname{Ann}_{R_{2}}\left(N_{2}\right)$ is a 2-absorbing primary ideal of $R_{2}$. Then $N_{1}=0$ and $N_{2}$ is a strongly $G 2$-absorbing second submodule of $M_{2}$ by Theorem 2.15 (a) and Lemma 2.22. Similarly if 
$\operatorname{Ann}_{R_{2}}\left(N_{2}\right)=R_{2}$ and $\operatorname{Ann}_{R_{1}}\left(N_{1}\right)$ is a 2-absorbing primary ideal of $R_{1}$. Then $N_{2}=0$ and $N_{1}$ is a strongly $G 2$-absorbing second submodule of $M_{1}$. If the last case hold, then as $M_{1}$ (resp. $M_{2}$ ) is a comultiplication $R_{1}$-(resp. $\left.R_{2^{-}}\right)$module, $N_{1}\left(\operatorname{resp} . N_{2}\right)$ is a secondary submodule of $M_{1}\left(\operatorname{resp} . M_{2}\right)$ by $[4,2.25]$.

$(b) \Rightarrow(a)$. This can be proved easily by using Theorem 2.23.

\section{References}

[1] Ansari-Toroghy H., Farshadifar F., Fully idempotent and coidempotent modules, Bull. Iranian Math., Soc. 38 (4), (2012), 987-1005.

[2] Ansari-Toroghy H., Farshadifar F., On the dual notion of prime submodules, Algebra Colloq., 19 (Spec 1), (2012), 1109-1116.

[3] Ansari-Toroghy H., Farshadifar F., Some generalizations of second submodules, Palestin J. Math., 8 (2), (2019), 159-168.

[4] Ansari-Toroghy H., Farshadifar F., 2-absorbing and strongly 2-absorbing secondary submodules of modules, Le Matematiche, 72 (11), (2017), 123-135.

[5] Ansari-Toroghy H., Farshadifar F., The dual notion of multiplication modules, Taiwanese J. Math., 11 (4), (2007), 1189-1201.

[6] Ansari-Toroghy H., Farshadifar F., On the dual notion of prime radicals of submodules, Asian Eur. J. Math., 6 (2), (2013), 1350024 (11 pages).

[7] Ansari-Toroghy H., Farshadifar F., Pourmortazavi S. S., On the P-interiors of submodules of Artinian modules, Hacet. J. Math. Stat., 45(3), (2016), 675-682.

[8] Ansari-Toroghy H., Farshadifar F., Pourmortazavi S.S., Khaliphe F. On secondary modules, Int. J. Algebra, 6 (16), (2012), 769-774.

[9] Badawi A., On 2-absorbing ideals of commutative rings, Bull. Austral. Math, Soc. 75, (2007), 417-429.

[10] Badawi A.,Tekir U.,Yetkin E., On 2-absorbing primary ideals in commutative rings, Bull. Korean Math, Soc. 51 (4), (2014), 1163-1173.

[11] Baig M., Primary Decomposition and Secondary Representation of Modules over a Commutative Ring, Thesis, Georgia State University, (2009).

[12] Ceken S., Alkan M., Smith P. F., The dual notion of the prime radical of a module, J. Algebra, 392, (2013), 265-275.

[13] Darani A. Y., Soheilnia F., 2-absorbing and weakly 2-absorbing submoduels, Thai J. Math., 9 (3), (2011), 577-584.

[14] Dauns J., Prime submodules, J. Reine Angew. Math., 298, (1978), 156-181.

[15] Fuchs L., Heinzer W., Olberding B., Commutative ideal theory without finiteness conditions: Irreducibility in the quotient filed, in : Abelian Groups, Rings, Modules, and Homological Algebra, Lect. Notes Pure Appl. Math., 249, (2006), 121-145.

[16] Payrovi Sh., Babaei S., On 2-absorbing submodules, Algebra Collq., 19, (2012), 913-920.

[17] Yassemi S., The dual notion of prime submodules, Arch. Math. (Brno), 37, (2001), 273-278.

[18] Yassemi S., The dual notion of the cyclic modules, Kobe. J. Math., 15, (1998), $41-46$. 


\section{CONTACT INFORMATION}

H. Ansari-Toroghy, Department of Pure Mathematics, Faculty of S. MalekiMathematical Sciences, University of Guilan, Roudposhti P.O. Box 41335-19141, Rasht, Iran E-Mail(s): ansari@guilan.ac.ir, Sepidehmaleki.r@gmail.com

F. Farshadifar

Department of Mathematics, Farhangian University, Tehran, Iran

E-Mail(s): f.farshadifar@cfu.ac.ir

Received by the editors: 06.12.2017. 